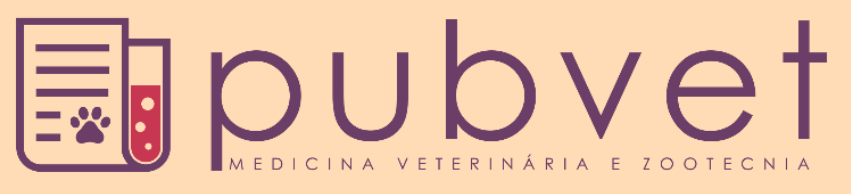

https://doi.org/10.31533/pubvet.v13n7a375.1-9

\title{
Descrição das categorias de comportamentos do Dasyprocta prymnolopha em condições de cativeiro
}

\author{
Jalison Figuerêdo do Rêgo ${ }^{\circ}$, Maria Acelina Martins de Carvalho' ${ }^{\circ}$, Ana Yasha \\ Ferreira de La Salles ${ }^{3} \bullet$, Danilo José Ayres de Menezes ${ }^{*} \bullet$
}

${ }^{I}$ Mestre em Psicobiologia, Universidade Federal do Rio Grande do Norte, Centro de Biociências, Natal, Rio Grande do Norte, Brasil. ${ }^{2}$ Professora do Departamento de Morfofisiologia Veterinária, Universidade Federal do Piauí, Centro de Ciências Agrárias, Teresina, Piauí, Brasil ${ }^{3}$ Aluna de Doutorado do Programa de Pós-Graduação em Ciência e Saúde Animal, Universidade Federal de Campina Grande, Patos, Paraíba, Brasil. ${ }^{4}$ Professor do Departamento de Ciências Biológicas, Universidade Federal do Rio Grande do Norte, Centro de Biociências, Natal, Rio Grande do Norte, Brasil. *Autor de correspondência, mdanayres@gmail.com

Resumo. Entre as espécies mais predadas, a cutia tem sido notada por ter sofrido uma redução significativa de suas populações, levando-as a serem frequientemente mantidas em cativeiro. O objetivo deste estudo foi descrever as categorias comportamentais da cutia em cativeiro, visando contribuir para o estudo de hipóteses sobre a biologia e o manejo desse roedor, bem como analisar a influência dos comportamentos com a sobrevivência e a reprodução das espécies cativas. A pesquisa foi realizada no Núcleo de Estudos e Preservação de Animais Silvestres (NEPAS) da Universidade Federal do Piauí. Foram observadas durante 1860 horas dois grupos de Dasyprocta prymnolopha, sendo o primeiro composto por dois machos, cinco fêmeas e quatro filhotes; e o segundo, por dois machos, sete fêmeas e um filhote, totalizando 21 animais pertencentes a grupos já formados e mantidos em cativeiro no NEPAS. O comportamento dos animais foi registrado manualmente (usando uma prancheta, lápis e cadernos) e, eventualmente, utilizou binóculos para observação detalhada de comportamentos isolados. Encontramos 14 categorias comportamentais, sendo capazes de identificar oito comportamentos distintos: comportamento alimentar; descansar; exploração; cuidados com o corpo; coleta e transporte de material; comportamentos agonísticos; comportamentos reprodutivos e marcação do território. Concluiu-se que as análises comportamentais apresentadas podem trazer contribuições práticas para a adequação das instalações dos animais, bem como a melhoria da gestão, melhorando o bem-estar das cutias em regime cativo.

Palavras-chave: Comportamento animal, etograma, cutia, Dasyproctidae

\section{Description of the Behaviors Categories of the Dasyprocta Prymnolopha in Conditions of Captivity}

\begin{abstract}
Among the most preyed species, the agouti has been noted for having suffered a significant reduction of their populations, leading to them being frequently kept in captivity. The objective of this study was to describe the behavioral categories of the agouti in captivity, aiming to contribute to the study of hypotheses about the biology and the management of this rodent, as well as to analyze the influence of the behaviors with the survival and reproduction of the captive species. The research was carried out at the Center for Studies and Preservation of Wild Animals (NEPAS) of the Federal University of Piauí. Were observed for 1860 hours two groups of Dasyprocta prymnolopha, the first consisting of two males, five females and four pups, and the second by two males, seven females and one cub, totalizing 21 animals belonging to groups already formed and kept in captivity in NEPAS. The behavior of the animals was recorded manually (using a drawing board,
\end{abstract}


pencils and notebooks) and eventually used binoculars for detailed observation of isolated behaviors. We found 14 behavioral categories, being able to identify eight distinct behaviors: food behavior; rest; exploration; body care; collection and transportation of material; agonistic behaviors; reproductive behaviors and marking of territory. It was concluded that the behavioral analyzes presented can bring practical contributions to the adequacy of the animal facilities, as well as the improvement of the management, improving the welfare of the agouti in captive regime.

Keywords: Animal behavior, ethogram, agouti, Dasyproctidae

\section{Descripción de las categorías de comportamiento de Dasyprocta prymnolopha en cautiverio}

Resumen. Entre las especies más capturadas, se ha observado que el Agutí ha reducido significativamente sus poblaciones, lo que las lleva a ser mantenidas frecuentemente en cautiverio. El objetivo de este estudio fue describir las categorías de comportamiento del agutí en cautiverio, con el objetivo de contribuir al estudio de las hipótesis sobre la biología y el manejo de este roedor, así como analizar la influencia de los comportamientos en la supervivencia y la reproducción de las especies cautivas. La investigación se realizó en el Centro de Estudios y Conservación de Animales Silvestres (NEPAS) de la Universidad Federal de Piauí. Se observaron dos grupos de Dasyprocta prymnolopha durante 1860 horas, el primero consistió en dos machos, cinco hembras y cuatro crías; y el segundo, por dos machos, siete hembras y una cría, con un total de 21 animales pertenecientes a grupos ya formados y mantenidos cautivos en NEPAS. El comportamiento de los animales se registró manualmente (utilizando un portapapeles, lápiz y cuadernos) y, finalmente, se usaron binoculares para la observación detallada de comportamientos aislados. Encontramos 14 categorías de comportamiento, pudiendo identificar ocho comportamientos distintos: comportamiento alimentario; descansar exploración; cuidado del cuerpo; recogida y transporte de material; conductas agonistas; Conductas reproductivas y marcaje del territorio. Se concluyó que los análisis de comportamiento presentados pueden aportar contribuciones prácticas a la adecuación de las instalaciones de los animales, así como a la mejora de la gestión, mejorando el bienestar de los agutíes en cautividad.

Palabras clave: Comportamiento animal, etograma, agutí, Dasyproctidae

\section{Introdução}

Estudo com animais silvestres proporciona grande utilidade no campo da agropecuária, comércio, indústria. Várias pesquisas realizadas nestas áreas, em entidades públicas e privadas, estão sendo desenvolvidas proporcionando a melhoria da qualidade de vida humana, socioeconômica, progresso da ciência, educação ambiental, estudos em bem-estar animal e enriquecimento ambiental.

Atualmente muitas espécies são criadas em cativeiro, dentre elas estão os mamíferos pertencentes à família Dasyproctidae. Esta família é constituída por roedores da subordem Hystricognathi, divididos em dois gêneros: Dasyprocta (as cutias) e Myoprocta (as cotiaras). Entre as espécies mais predadas, a cutia tem se destacado por ter sofrido significativa redução de suas populações, tanto pela caça predatória como pela destruição de seu habitat natural (Hosken \& Silveira, 2001). A criação destes animais em cativeiro pode vir como uma solução para esse problema, mantendo a espécie selvagem salva da predação.

As cutias distribuem-se geograficamente desde a América do Sul até América Central (Eisenberg, 1989), sendo os machos normalmente menores que as fêmeas. Os membros pélvicos são bem maiores do que os torácicos, sendo utilizados para o salto (Hosken \& Silveira, 2001). Considerados mamíferos exclusivamente fitófagos, alimentam-se geralmente de frutos, raízes e vegetais suculentos, além de sementes de diversas árvores que encontra na natureza, caracterizando a importância destes animais nos processos de dispersão de sementes (Galetti et al., 2010; Gorchov et al., 2004; Pimentel \& Tabarelli, 2004). Tem como habitat as florestas pluviais (floresta amazônica e mata atlântica), florestas 
semidecíduas, cerrados e caatingas, geralmente com a distribuição associada a cursos de água (Organização Pan-Americana da Saúde, 2008).

O conhecimento das particularidades ecológicas e comportamentais das cutias facilita sua criação. Determinados comportamentos podem ser alterados, quando se trata de animais silvestres mantidos em cativeiro, considerando seu manejo dificultado caso não haja conhecimento, principalmente, de sua reprodução aliada aos seus hábitos cotidianos.

Diante disso, o presente trabalho tem como principal objetivo a contribuição para o conhecimento do comportamento da cutia em regime de cativeiro, cujos resultados poderão servir de base para melhoria do manejo da espécie, bem como, entendimento sobre a sua biologia extrapolando para o comportamento no seu habitat natural, assim como analisar a influência dos comportamentos com a sobrevivência e a reprodução da espécie em cativeiro.

\section{Material e métodos}

A pesquisa foi realizada no Núcleo de Estudos e Preservação de Animais Silvestres (NEPAS) do Centro de Ciências Agrárias da Universidade Federal do Piauí. Implantado desde maio de 1998, o NEPAS desenvolve estudos com animais silvestres, procurando explorar seus potenciais econômicos. Os trabalhos são diversificados, variando desde análise anatômica, sanidade, genética e citogenética, anestesiologia, até estudos relacionados com desmame de filhotes e obtenção de ração balanceada, com custo baixo, para produção e comercialização para ambiente de cativeiro.

Foram selecionados grupos de animais já formados, que vivem em cativeiro nas baias do Núcleo de Animais Silvestres, não havendo mudanças no espaço físico e na quantidade de cutias distribuídas em cada baia.

O comportamento dos animais foi registrado de forma manual (com utilização de prancheta, lápis e fichas de anotações). Eventualmente utilizou-se de binóculo para observação detalhada de comportamentos isolados. As observações foram realizadas utilizando o método de varredura, com registro instantâneo das categorias comportamentais.

Foram utilizados 2 boxes/piquetes de $26,3 \mathrm{~m}^{2}$ cada, O primeiro contendo dois machos, cinco fêmeas e quatro filhotes e o segundo possuindo dois machos, sete fêmeas e um filhote.

Os animais foram observados durante um ano, totalizando 1.860 horas de observação. O registro das descrições comportamentais realizou-se semanalmente (exceto sábado e domingo), pelo período da manhã, entre as 08:00h e as 11:00h, e a tarde, entre as 14:00h e 17:00h. A escolha do horário de observação foi baseada na pesquisa de Emmons \& Feer (1997), que identifica esses períodos como aqueles em que ocorrem picos de atividade das cutias (início da manhã ou final da tarde), assim como no período de descanso (próximo ao meio-dia ou início da tarde).

\section{Resultados e discussão}

Foram descritas 12 categorias comportamentais, podendo destacar oito comportamentos distintos: descanso, exploração, comportamento alimentar, cuidados corporais, coleta e transporte de material, comportamentos agonísticos, comportamentos reprodutivos e marcação de território.

\section{Descanso}

Foi possível caracterizar nove principais posições e/ou apoio de partes do corpo no local de descanso, representado por uma toca ou refúgio:

1. Membros encolhidos, corpo no chão e cabeça deitada no chão, com olhos levemente fechados.

2. Corpo deitado com membros pélvicos e torácicos estirados.

3. Corpo deitado com membros pélvicos encolhidas e torácicos estirados.

4. Posição sentada, com patas traseiras servindo de base.

5. Parte do corpo deitado, com uma pata traseira e dianteira encolhida; e as outras estiradas.

6. Acompanhado de outros (no total de três animais juntos).

7. Parte do corpo deitado, e patas traseiras e dianteiras estiradas. 
8. Corpo encolhido, inclinado um pouco de lado, formando uma leve curvatura e apenas uma pata traseira (esquerda), estirada.

9. Sobre um tronco de árvore e corpo deitado de lado e estirado.

Estas posturas são semelhantes às observadas por Kraus et al. (1970), em suas pesquisas com rodentia. Santos (2005) estudando a ecologia de Dasyprocta leporina em um fragmento florestal, observou que esses animais dedicavam parte do tempo para descansar, principalmente no final da manhã e início da tarde, onde o comportamento deitado foi registrado com 15,06\% de frequência.

\section{Exploração}

A exploração desses animais se dá, em grande parte, por meio do farejamento. A cutia costuma farejar o chão ou o ar (levantando o focinho para cima), em sentido reto ou lateral, nos dois sentidos da cabeça. Ao farejar, locomove-se constantemente, chegando a apoiar-se com os membros torácicos nas paredes das baias, semelhante ao que foi observado nas pacas criadas em cativeiro (Sabatini \& Paranhos da Costa, 2001). Entretanto, quando fareja o piso, a cabeça fica em posição normal em relação à postura do animal. A cutia foi observada várias vezes, pulando em cima da mureta, explorando por toda extensão da grade de proteção.

Santos (2005) relatou em pesquisa com Dasyprocta leporina, que o comportamento de farejar foi o que apresentou o maior número de ocorrências, correspondendo a 36,9\%. O mesmo autor relacionou esse comportamento ao olfato aguçado desses animais, e à busca pela recuperação de sementes enterradas por eles mesmos em épocas de maior disponibilidade de alimentos. Além disso, é relatada a utilização do olfato como meio de comunicação com os demais animais do grupo (Emmons \& Feer, 1997).

\section{Comportamento Alimentar}

\section{a. Carregar, enterrar e desenterrar alimento}

Alimentos industrializados, como é o caso da ração fornecida eram digeridos pelas cutias no local onde eram colocados (tanque de cimento apropriado para alimentação), podendo, em várias ocasiões, algumas pequenas quantidades isoladas, serem removidas e carregadas para outros locais do recinto. Quando colocadas frutas e verduras, era comum a retirada do alimento, com a boca, do local onde era inicialmente colocado. As cutias carregam normalmente estes alimentos para locais isolados da baia, procurando digerir sem a interferência de membros do grupo. $\mathrm{O}$ mesmo comportamento pode ser observado na natureza por alguns gêneros de histricognatos, os quais carregam o alimento para locais específicos, preferindo as partes mais escuras da mata (Smythe, 1983). Frutos de palmeira pertencentes ao gênero Orbignya sp (conhecido popularmente como babaçu) eram carregados com dentes inseridos nas extremidades, um por vez, para cada local da baia. A ração granulada, dificilmente foi enterrada. Entretanto, foram observados alguns grãos isolados sendo enterrados por qualquer membro do grupo. A cutia retirava o grão do local de alimentação, segurando com os dentes e levava para locais aleatórios da baia para enterrar. Cavava o chão com as mãos, soltando o grão posteriormente, e o enterrando. Outros alimentos, também foram enterrados pelas cutias, como sementes, verduras e frutas. Foram observados alimentos sendo parcial ou totalmente enterrados. Essa característica de enterrar o alimento é muito observada na natureza em diferentes espécies, sejam nas próprias cutias, cutiaras, marsupiais, roedores (Forget et al., 2001), macacos, e até de elefantes (Chapman \& Chapman, 1995). Na pesquisa de Silvius (2002), o autor relata que as cutias não moveram sementes para longe do seu local de origem. Para o autor, este comportamento explica o papel das cutias como dispersoras de sementes a distâncias extremamente curtas. Esse fato se explica mediante diversos estudos, os quais relatam que cutias geralmente alimentam-se de sementes acumuladas que desenterram no limite de cinco metros da plantamãe ou estação experimental de sementes (Peres \& Baider, 1997; Silva \& Tabarelli, 2001), apesar de que elas ocasionalmente carregam sementes por vinte, trinta e até duzentos metros da fonte, em algumas ocasiões (Peres et al., 1997).

\section{b. Segurar com os membros torácicos}

Com a cutia em posição sentada, todos os alimentos (por menor que fossem) eram segurados pelas mãos antes de serem ingeridos, sendo levados até próximo à boca. Este hábito de usar os membros na alimentação é bastante comum entre os roedores e foi bastante observado por Sabatini \& Paranhos da 
Costa (2001), em suas pesquisas com pacas. Kaiser et al. (2011) relataram em estudo de comportamento em cativeiro com as espécies Dasyprocta azarae e Dasyprocta leporina, que durante a alimentação, o alimento foi manipulado com os membros torácicos, transportado e ingerido. Contudo, registrou-se uma cutia fêmea na segunda baia alimentando-se, deitada dentro do local de alimentação, sem auxílio das mãos, o que não foi encontrado em nenhum experimento relatado na literatura.

\section{c. Descascar}

É um comportamento bem característico das cutias, o qual foi registrado somente com grãos e frutos secos. Nos grãos de milho, estes roedores possuem o hábito de retirar as películas que envolvem as sementes, no entanto, essa atividade só é possível quando os grãos estão bastante úmidos ou molhados. Ades \& Busch (2000) relataram que esse tipo de comportamento pode ser modificado de acordo com o desempenho proporcionado pelo animal e a atividade costuma-se ser mais eficiente à medida que o animal a repete.

Já frutos secos, são descascados sem a necessidade de serem molhados, retirando as cascas com auxílio das mãos e dentes. Ocasionalmente estes roedores carregavam, com os dentes, frutos de babaçu até o bebedouro, soltando-os posteriormente dentro d'água. Após alguns minutos retornava ao local do bebedouro, mexendo o fruto como se estivesse lavando-o e depois o removia da água. $\mathrm{O}$ ato de molhar os frutos podia ser repetido outras vezes antes de retirá-lo definitivamente da água e roê-lo.

$\mathrm{Na}$ manipulação de itens alimentares por roedores são encontrados vários exemplos. O comportamento do descascamento de sementes pelo camundongo Calomys callosus, foi estudado por Ades \& Busch (2000). Em pesquisa com esquilos (Sciurus vulgaris) criados em cativeiro, observou-se que a repetição do mecanismo de descascar avelãs, foi se tornando eficiente com o número de tentativas, em indivíduos sem experiência prévia (Eibl-Eibesfeldt, 1956). Em outro estudo com ratos (Rattus norvegicus), foi observado que esses animais modificaram, ao longo de um contato repetido, a maneira como abriam avelãs, e que essa experiência era transferida para outros itens alimentares (Kemble et al., 1983).

\section{d. Disputa por alimentos}

Quando o animal está se alimentando, é frequente a aproximação de um indivíduo na tentativa de roubar o alimento. Após bem-sucedida retirada do alimento, o outro animal pode tentar recuperá-lo ou não. As tentativas de disputar alimentos com outros indivíduos e as possíveis tentativas de recuperação foram registradas em pesquisas feitas com Agouti paca em cativeiro (Sabatini \& Paranhos da Costa, 2001). A retirada do alimento pode ocorrer em relação à dominância ou ocasional roubo investido por jovens em adultos (comportamento parental e afiliativo). Rodrigues et al. (2013) relataram em estudo realizado com Capivaras, conflitos entre os animais estudados, principalmente por disputas por alimentos e geralmente entre o macho dominante e machos submissos, igualmente ao relatado nessa pesquisa.

\section{e. Preferência alimentar}

A ração é a principal refeição, sendo complementadas com grãos de milho, cascas de verduras e frutos secos. Observou-se a mastigação de folhas e flores em tamanhos variados, quando estas caiam dentro do recinto. Frutos carnosos podem ser deglutidos, uma vez colocados nas baias. Em suas pesquisas, Smythe (1983) e Nowak \& Paradiso (1983) descreveram que cutias se alimentam principalmente de frutos, sementes e diversas plantas suculentas. Semelhantes preferências alimentares são descritas por Moojen (1952), Smythe (1983) e Pinheiro et al. (1989). Outro autor relata que cutias se alimentam de sementes quando há escassez de frutos (Henry, 1999). Esta preferência alimentar ainda pode ser enriquecida por folhas, flores e cogumelos (Emmons \& Feer, 1997).

Insetos que caem no local de alimentação podem ser atacados e digeridos pelas cutias, como cupins, besouros, borboletas e larvas. Entretanto, os insetos que entraram nas baias, sem caírem por acidente, foram ignorados e não ocorreu predação por parte dos animais. Foram encontradas estruturas dilaceradas de animais semelhantes a lagartos e aves. Contudo, não foi observado o ataque das cutias nestes animais. Constantemente notou-se a entrada de pássaros e lagartos dentro das baias, sem nenhum ataque investido pelas cutias. Contradizendo o observado nesse estudo; Monteiro-Filho et al. (1999), em pesquisa com Dasyprocta azarae em cativeiro, visando verificar se a predação de ovos e aves seria um comportamento inato ou aprendido para a espécie, observou que esses animais apresentaram pronta predação, tanto dos ovos, quanto das aves, mesmo nunca tendo tido contato com esse tipo de alimento. 


\section{f. Cecotrofia}

O fenômeno da cecotrofagia consiste na ingestão de um tipo especial de fezes (cecotrofo) que são eliminados por contrações específicas do ceco (Pinheiro et al., 2001). Estudos já realizados com capivaras e coelhos demonstram que esses animais diferem o cecotrofo das fezes normais, e excretam dois tipos de fezes, uma oval na forma de pellets individualizada de coloração verde-oliva e outra de consistência pastosa e coloração mais clara. Esta última contém em média $37 \%$ a mais de proteína do que as fezes normais (Mendes et al., 2000). Esse mecanismo fisiológico permite uma nova assimilação de nutrientes, especialmente aminoácidos, bem como o aporte extra de proteínas e vitaminas (Pinheiro et al., 2001). O comportamento de cecotrofagia é específico de lagomorfos, pequenos roedores, capivaras e alguns outros mamíferos (Caroline et al., 2003), sendo observado em herbívoros como decorrência da adaptação do processo digestivo às difíceis condições ambientais, particularmente alimentares, o que permite a reciclagem de parte do alimento não digerido, possibilitando sua melhor utilização. Algumas cutias demonstraram aproximação e deglutição de fezes, durante o período de alimentação. Contudo, esta ação comportamental pareceu ser menos evidente nestes animais. A deglutição de fezes ocorreu, porque as fezes estavam juntas a alguns grãos de ração.

\section{Marcação}

A cutia mantém contato com o chão, por meio da região perineal, enquanto enterra alimentos e em algumas ocasiões esfrega a própria região perineal no local aonde teria enterrado sementes. Este comportamento possivelmente é utilizado para marcação de odor no local aonde o alimento foi enterrado (Santos, 2005).

Howard et al. (1968) constataram que roedores do gênero Peromyscus podem sentir o cheiro de sementes enterradas nos locais aonde realiza exploração. Contudo, são necessários mais estudos para comprovação da utilização da marcação de cheiro na localização de alimentos enterrados pelas cutias. Em outra situação, Santos (2005), em estudo de comportamento da espécie Dasyprocta leporina, relatou que a comunicação olfativa desses animais é realizada através de odores deixados pela secreção de uma glândula anal e pela urina, e esses odores atuam como delimitadores territoriais para localizarem o alimento anteriormente escondido e na identificação de membros do mesmo grupo. Em experimento com capivaras em cativeiro, as marcações de território foram observadas apenas durante a noite, e na maioria das vezes, pela glândula paranal em pequenos arbustos (Rodrigues et al., 2013).

\section{Cuidados corporais}

Esses animais promovem a limpeza de partes do corpo regularmente com a língua, sem muitas repetições. Os dentes são utilizados na procura e retirada de parasitas (autolimpeza). Esta limpeza corporal e retirada de parasitas pode ser realizada em outros indivíduos, não importando a condição de hierarquia. Condições semelhantes já foram descritas anteriormente, as quais foram denominadas respectivamente de autogrooming e alogrooming (Smythe, 1983). Esse tipo de comportamento, chamado popularmente de catação, são comuns em primatas, sendo muito importante dentro dos grupos, e usualmente direcionado ao macho ou fêmea dominante (Jablonski Júnior, 1988). Coçar com os membros torácicos sobre o corpo, não foi observado frequentemente, ocorrendo geralmente em situações de descanso ou repouso, tendo certa semelhança ao observado por Sabatini \& Paranhos da Costa (2001) em Agouti paca. O mesmo autor relata a observação de outros cuidados corporais nesta espécie, como esfregar o focinho, lamber-se e secar-se, o que não foi observado nos animais do estudo.

\section{Coleta e transporte de material}

Atividade pouco registrada, trata-se do comportamento de forragem no local aonde descansa após refeições e atividades do dia. A cutia abocanha folhas, fibras secas, vegetais e palhas encontradas na baia e leva para o local de descanso, procurando forrar o ambiente em que dorme e descansa. Rosenbaum et al. (2009), em seus estudos com roedores, relataM que a preferência pelo tipo de material utilizado como cama pode variar bastante, não apenas em relação ao material, mas também à forma como este é disponibilizado. Kraus et al. (1970) realizando pesquisas com rodentia, resgistram que a coleta de material é realizada somente pela boca e as mãos não são usadas neste contexto comportamental. Nossas análises são semelhantes a esta observação constatada anteriormente, sendo esta coleta normalmente individual, ocorrendo de forma aleatoria e pouco frequente. 


\section{Comportamentos agonísticos}

Em decorrência de alguns sinais de perigo (como por exemplo, indivíduos desconhecidos, sons fortes e estranhos), as cutias batem rapidamente os membros pélvicos no chão. Este comportamento que é denominado de thumping (Smythe, 1983) pode ser seguido pela emissão de gritos ou sons mecânicos (com os dentes). Em outras ocasiões, levanta a cabeça em posição imóvel e em sinal de alerta, podendo realizar corridas rápidas e aleatórias ou eriçar os pelos dorsais, caso se sinta bastante ameaçada. Lima (2018), em estudo realizado com as espécies Cuniculus paca e Dasyprocta leporina, sobre comportamento acústico, observou comportamento de thumping durante a separação mãe/fillhote. Além deste, foram relatados emissão vocal de "purr" - sugerindo um chamado de contato, batidas de dente e rosnados. Tais características destes comportamentos foram também observadas por Smythe (1983), tanto na natureza, como em cativeiro, na espécie Dasyprocta punctata.

Nos dois grupos observados, machos raramente realizaram agressões entre eles. As fêmeas, porém, eram mais agressivas entre si e constantemente defendiam seus alimentos, locais de descanso e exploração. Spotorno et al. (2004) relatam que as Chinchilas (Chinchilla lanigera) fêmeas também são mais agressivas que os machos quanto à defesa territorial. Smythe (1983) descreveu estes comportamentos hostis das fêmeas de Dasyprocta punctata somente na natureza. No entanto, relações agonísticas entre fêmeas de Agouti paca em regime de cativeiro foi constatado por Alho \& Cleber (1986) durante suas análises. Estes movimentos de perseguições e correrias foram muito frequentes durante as observações. Fêmeas dominantes realizam perseguições em fêmeas submissas, podendo até investir perseguições em machos.

\section{Comportamento reprodutivo}

Este comportamento não foi visto com frequência durante o período de observação, apesar de ser relatado em estudo, com Cuniculus paca e Dasyprocta leporina, que há evidências de que fatores de ordem reprodutiva, como a seleção sexual, podem levar ao aumento na complexidade acústica das espécies (Freeberg et al., 2012).

Os machos normalmente iniciam comportamento de corte com ereção do pênis, seguido de eliminação de urina, tremor nos membros torácicos e investidas de perseguições à fêmea, com tentativas de monta. Durante o acasalamento, machos podem realizar movimentos de caminhada de forma ondulatória, como fossem rituais de dança. Smythe (1983) caracterizou este comportamento como frenzy dance, a qual é executada durante o ritual de corte e cópula na Dasyprocta punctata. Pollard \& Blumstein (2012) relatam que o aumento no tamanho do repertório vocal de roedores, usado para atrair ou impressionar parceiros sexuais, é variável de acordo com a complexidade do sistema de acasalamento.

\section{Conclusão}

As cutias apresentaram uma variedade de comportamentos, sendo alguns dependentes da oferta de recursos e de como estes recursos eram oferecidos. Além disso, alguns contextos comportamentais apresentados nos animais em cativeiro foram semelhantes àqueles na natureza, o que demonstra o comportamento inato frente a certas situações. Em decorrência, é perceptível que as análises do repertório comportamental apresentado, podem trazer contribuições práticas para a adequação das instalações dos animais, assim como a melhoria do manejo, melhorando o bem-estar das cutias em regime de cativeiro.

\section{Agradecimentos}

A Prof. Dr ${ }^{\text {a }}$ Selene S. da Cunha Nogueira por ter cedido gentilmente o material bibliográfico;

A coordenação do NEPAS da Universidade Federal do Piauí, pelo uso de suas instalações, quando das observações com os animais.

\section{Referências bibliográficas}

Ades, C. \& Busch, S. E. (2000). A aprendizagem do descascamento de sementes pelo camundongo Calomys callosus (Rodentia, Cricetidae). Revista Brasileira de Zoociências, 2(1):31-44. 
Alho, C. J. R. \& Cleber, J. (1986). Criação e manejo de capivaras em pequenas propriedades rurais (Vol. 1). Brasília, Brasil: EMBRAPA.

Caroline, J., Gross, M. S. \& Spillman, D.-M. (2003). Fiber digestion in mammals. Pak. J. Biol. Sci, 6(17):1564-1573.

Chapman, C. A. \& Chapman, L. J. (1995). Survival without dispersers: seedling recruitment under parents. Conservation Biology, 9(3):675-678.

Eibl-Eibesfeldt, I. (1956). Über die ontogenetische Entwicklung der Technik des Nüsseöffnens vom Eichhörnchen. Zeitschrift für Säugetierkunde, 21132-134.

Eisenberg, J. F. (1989). Mammals of the Neotropics, (v. 1) The northern neotropics: Panama, Colombia, Venezuela, Guyanna, Suriname, French Guiana. Chicago, USA: Univerty of Chicago Press.

Emmons, L. H. \& Feer, F. (1997). Neotropical rainforest mammals: A Field Guide. Chicago, USA: The University of Chicago Press.

Forget, P. M., Hammond, D. S., Milleron, T. \& Thomas, R. (2001). Seasonality of fruiting and food hoarding by rodents. In D. J. Levey, W. R. Silva \& M. Galetti (Eds.), Seed dispersal and frugivory: ecology, evolution and conservation. Wallingford, England: CABI.

Freeberg, T. M., Ord, T. J. \& Dunbar, R. I. M. (2012). The social network and communicative complexity: preface to theme issue. Philosophy Transactions Royal Society, 367(1782-1784).

Galetti, M., Donatti, C. I., Steffler, C., Genini, J., Bovendorp, R. S. \& Fleury, M. (2010). The role of seed mass on the caching decision by agoutis, Dasyprocta leporina (Rodentia: Agoutidae). Zoologia, 27(3).

Gorchov, D. L., Palmeirim, J. M. \& Ascorra, C. F. (2004). Dispersal of seeds of Hymenaea courbaril (Fabaceae) in a logged rain forest in the Peruvian Amazonian. Acta Amazonica, 34(2):251-259.

Henry, O. (1999). Frugivory and the importance of seeds in the diet of the orange-rumped agouti (Dasyprocta leporina) in French Guiana. Journal of Tropical Ecology, 15(3):291-300.

Hosken, F. M. \& Silveira, A. C. (2001). Criação de cutias. Viçosa, Minas Gerais, Brasil.: Aprenda Fácil.

Howard, W. E., Marsh, R. E. \& Cole, R. E. (1968). Food detection by deer mice using olfactory rather than visual cues. Animal Behaviour, 1613-18.

Jablonski Júnior, S. J. (1988). Análise da interação social de machos e fêmeas em duas espécies de saguis (Callithrix jacchus e Callithrix penicillata). Master of Science, Universidade Federal de Santa Catarina, Florianópolis, Santa Catarina, Brasil.

Kaiser, S. K., Margarido, T. C. C. \& Fischer, M. L. (2011). Avaliação do comportamento de cutias Dasyprocta azarae e Dasyprocta leporina (Rodentia: Dasyproctidae) em cativeiro e semicativeiro em parques urbanos de Curitiba, Paraná, Brasil. Revista de Etologia, 10(2):68-82.

Kemble, E. D., Wimmer, S. C. \& Konkler, A. P. (1983). Effects of varied prior manipulatory or consummatory behaviours on nut opening, predation, novel foods consumption, nest building, and food tablet grasping in rats. Behavioural Processes, 8(1):33-44.

Kraus, C., Gihr, M. \& Pilleri, G. (1970). Das verhalten von Cuniculus paca (Rodentia, Dasyproctidae) in gefangenschaft. Revue Suisse de Zoologie, 77353-388.

Lima S. G. C, Sousa-Lima R. S, Tokumaru R.S, Nogueira-Filho S. L. G. \& Nogueira S. S. C. (2018) Vocal complexity and sociality in spotted paca (Cuniculus paca). PLoS ONE 13(1), e0190961.

Mendes, A., Nogueira, S. S. C., Lavorenti, A. \& Nogueira-Filho, S. L. G. (2000). A note on the cecotrophy behavior in capybara (Hydrochaeris hydrochaeris). Applied Animal Behaviour Science, 66(1-2):161-167.

Monteiro-Filho, E. L. A., Margarido, T. C. C., Pachaly, J. R., Mangini, P. R., Montiani-Ferreira, F. \& Lange, R. R. (1999). Comportamento zoofágico inato de cutias-Dasyprocta azarae Lichtenstein, 1823 (Rodentia: Mammalia). Arquivos de Ciências Veterinárias e Zoologia da UNIPAR, 2(2):135142.

Moojen, J. a. (1952). Os roedores do Brasil. Rio de Janeiro, Brasil: Ministério da Educação e Saúde, Instituto Nacional do Livro, Biblioteca Científica Brasileira, série A - II. 
Nowak, R. M. \& Paradiso, J. L. (1983). Walker's mammals of the world. Baltimore, USA: The Johns Hopkins University Press.

Organização Pan-Americana da Saúde. (2008). Guia dos roedores do Brasil, com chaves para gêneros baseadas em caracteres externos. Rio de Janeiro, Brasil.

Peres, C. A. \& Baider, C. (1997). Seed dispersal, spatial distribution and population structure of Brazilnut trees (Bertholletia excelsa) in southeastern Amazonia. Journal of Tropical Ecology, 13(4):595-616.

Peres, C. A., Schiesari, L. C. \& Dias-Leme, C. L. (1997). Vertebrate predation of Brazil-nuts (Bertholletia excelsa, Lecythidaceae), an agouti-dispersed Amazonian seed crop: a test of the escape hypothesis. Journal of Tropical Ecology, 13(1):69-79.

Pimentel, D. S. \& Tabarelli, M. (2004). Seed dispersal of the palm Attalea oleifera in a remnant of the Brazilian Atlantic Forest. Biotropica, 36(1):74-84.

Pinheiro, M. J. P., Andrade, S. A. \& Cunha, J. N. (1989). Preservação e exploração de animais silvestres nativos: preá, cutia e mocó. Revista Caatinga, 6(1):28-49.

Pinheiro, M. S., Silva, J. J. C. \& Rodrigues, R. C. (2001). Utilização sustentável e domesticação da capivara. Pelotas, Rio Grande do Sul, Brasil.

Pollard, K. A. \& Blumstein, D. T. (2012). Evolving communicative complexity: insights from rodents and beyond. Philosophical Transactions of the Royal Society B: Biological Sciences, 367(1597):1869-1878.

Rodrigues, M. V., Paula, T. A. R., Ferreira, L. B. C., Ávila, E. C., Silva, L. C. \& Souza, V. B. (2013). Comportamento de um grupo de capivaras em uma área urbanizada. Acta Veterinaria Brasilica, 7(3):212-217.

Rosenbaum, M. D., VandeWoude, S. \& Johnson, T. E. (2009). Effects of cage-change frequency and bedding volume on mice and their microenvironment. Journal of the American Association for Laboratory Animal Science, 48(6):763-773.

Sabatini, V. \& Paranhos da Costa, M. J. R. (2001). Etograma da paca (Agouti paca, Linnaeus, 1766) em cativeiro. Revista de Etologia, 3(1):3-14.

Santos, E. F. (2005). Ecologia da cutia Dasyprocta leporina (Linnaeus, 1758) em um fragmento florestal urbano em Campinas - SP (rodentia: dasyproctidae). $\mathrm{PhD}$, Universidade Estadual Paulista, Rio Claro, São Paulo, Brasil.

Silva, M. G. \& Tabarelli, M. (2001). Seed dispersal, plant recruitment and spatial distribution of Bactris acanthocarpa Martius (Arecaceae) in a remnant of Atlantic forest in northeast Brazil. Acta Oecologica, 22(5-6):259-268.

Silvius, K. M. (2002). Spatio-temporal patterns of palm endocarp use by three Amazonian forest mammals: granivory or 'grubivory'? Journal of Tropical Ecology, 18(5):707-723.

Smythe, N. (1983). Dasyprocta punctata and Agouti paca. In D. H. Jansen (Ed.), Costa Rican natural history. Chicago, USA: The University of Chicago Press.

Spotorno, A. E., Valladares, J. P., Marín, J. C. \& Zeballos, H. (2004). Molecular diversity among domestic guinea-pigs (Cavia porcellus) and their close phylogenetic relationship with the Andean wild species Cavia tschudii. Revista Chilena de Historia Natural, 77(2):243-250.

Recebido: 1 de junho, 2019.

Aprovado: 1 de julho, 2019.

Publicado: 7 de agosto, 2019.

Licenciamento: Este artigo é publicado na modalidade Acesso Aberto sob a licença Creative Commons Atribuição 4.0 (CC-BY 4.0), a qual permite uso irrestrito, distribuição, reprodução em qualquer meio, desde que o autor e a fonte sejam devidamente creditados. 